\title{
On the numerical investigations to the Cahn-Allen equation by using finite difference method
}

\author{
Asıf Yokuş $^{a *}$, Hasan Bulut ${ }^{b}$ \\ ${ }^{a}$ Department of Actuary, Faculty of Science, Firat University, Elazig, Turkey \\ ${ }^{b}$ Department of Mathematics, Faculty of Science, Firat University, Elazig, Turkey \\ asfyokus@yahoo.com,hbulut@firat.edu.tr
}

\section{ARTICLE INFO}

Article history:

Received: 6 November 2017

Accepted: 23 January 2018

Available Online: 15 October 2018

Keywords:

Cahn-Allen equation

Finite Difference Method

Numerical Solution

AMS Classification 2010:

65L12, $74 S 20$

\begin{abstract}
In this study, by using the finite difference method (FDM for short) and operators, the discretized Cahn-Allen equation is obtained. New initial condition for the Cahn-Allen equation is introduced, considering the analytical solution given in Application of the modified exponential function method to the Cahn-Allen equation, AIP Conference Proceedings 1798, 020033 [1]. It is shown that the FDM is stable for the usage of the Fourier-Von Neumann technique. Accuracy of the method is analyzed in terms of the errors in $L_{2}$ and $L_{\infty}$. Furthermore, the FDM

is treated in order to obtain the numerical results and to construct a table including numerical and exact solutions as well as absolute measuring error. A comparison between the numerical and the exact solutions is supported with two and three dimensional graphics via Wolfram Mathematica 11.
\end{abstract}

(cc) $\mathbf{E Y}$

\section{Introduction}

Russel has firstly studied the solitary wave $[2,4]$ by following the water wave travelling through a tube. Investigation of the analytical and numerical solutions as well as other studies to the various class of nonlinear partial differential equations play an important role in the field of nonlinear sciences.

Most recently, some serious methods have been developed in order to solve nonlinear differential equation. For example, $\left(\mathrm{G}^{\prime} / \mathrm{G}\right)$-expansion method $[5,6]$, the improved $\left(G^{\prime} / G\right)$-expansion method [7-9], the modified simple equation method [10], the Sumudu transform method [11-14], the Bäcklund transform method [15], the homotopy analysis method [16,17], the exponential function method [18-20], the modified exponential function method [21], generalized Bernoulli sub-ODE method [22], improved Bernoulli sub-ODE method [24-26], weak solutions[27] and galerkin method [28].

In the current work, we consider the Cahn-Allen equation given as:

$$
u_{t}=u_{x x}-u^{3}+u \text {. }
$$

By using first integral method, Bulut et al. [23] have obtained some soliton to Eq. (1).
The discretize equation to the Cahn-Allen equation is derived by using the finite difference method (FDM) and its operators. We observe that the numerical method is stable with the Eq. (1) is stable when the Fourier-Von Neumann technique is utilzed. Furthermore, the accuracy in terms of the errors in and is analyzed. We then utilized the FDM in approximating exact and numerical solutions to Eq. (1). We present the computed exact and numerical approximations as well as the absolute error in tables. We compare the exact and numerical approximations calculated and support the comparison with some graphics plots, which are sketched by using the Wolfram Mathematica 11.

\section{Fundamental properties of methods}

\subsection{Analysis of FDM}

Some important notations are needed in order to describe the finite forward difference method, these are:

- $\Delta x$, which is the spatial step

- $\Delta t$, which is the time step

\footnotetext{
*Corresponding author
} 
- $x_{i}=a+i \Delta x, i=0,1,2, \ldots, N$ points, which are the coordinates of mesh and $N=\frac{b-a}{\Delta x}, t_{j}=j \Delta t, j=0,1,2, \ldots, M$ and $M=\frac{T}{\Delta t}$.

- The function $u(x, t)$ is the value of the solution at $u\left(x_{i}, t_{j}\right) \cong u_{i, j}$ (grid points), where $u_{i, j}$ will is the numerical approximations of the exact value of $u(x, t)$ at the points $\left(x_{i}, t_{j}\right)$.

The difference operators are given as follows:

$$
\begin{aligned}
& H_{t} u_{i, j}=u_{i, j+1}-u_{i, j}, \\
& H_{x x} u_{i, j}=u_{i+1, j}-2 u_{i, j}+u_{i-1, j} .
\end{aligned}
$$

Thus, the derivatives involve in Eq. (1) can be given in finite difference operators form as

$$
\begin{aligned}
& \left.\frac{\partial u}{\partial t}\right|_{i, j}=\frac{H_{t} u_{i, j}}{\Delta t}+O\left(\Delta t^{2}\right), \\
& \left.\frac{\partial^{2} u}{\partial x^{2}}\right|_{i, j}=\frac{H_{x x} u_{i, j}}{(\Delta x)^{2}}+O\left(\Delta x^{2}\right) .
\end{aligned}
$$

The difference operator form to Eq. (1) is given as

$$
\frac{H_{t} u_{i, j}}{\Delta t}=\frac{H_{x x} u_{i, j}}{(\Delta x)^{2}}-\left(u_{i, j}\right)^{3}+u_{i, j} .
$$

Inserting Eq. (4) and (5) into Eq. (1), one can be written as indexed

$$
\begin{aligned}
u_{i+1, j}= & -u_{i-1, j}+u_{i, j}\left(2-(\Delta x)^{2}-\frac{(\Delta x)^{2}}{\Delta t}\right) \\
& +(\Delta x)^{2}\left(u_{i, j}\right)^{3}+\frac{(\Delta x)^{2}}{\Delta t} u_{i, j+1},
\end{aligned}
$$

where the initial values $u_{i, 0}=u_{0}\left(x_{i}\right)$.

\subsection{Consistency analysis}

In this subsection, the consistency of Eq. (1) with difference method is discussed. Firstly, the Taylor series expansions as taking the following form [11-13],

$u_{i, j+1}=u_{i, j}+\Delta t \frac{\partial u}{\partial t}+O(\Delta t)^{2}$,

$u_{i-1, j}=u_{i, j}-\Delta x \frac{\partial u}{\partial x}+(\Delta x)^{2} \frac{\partial^{2} u}{\partial x^{2}}-O\left(\Delta x^{3}\right)$.

One may define the operator $L$ as

$$
L=\frac{\partial}{\partial t}-\frac{\partial^{2}}{\partial x^{2}} .
$$

The indexed form of operator $L$ takes the following form:

$$
L_{i, j}=\frac{H_{t} u_{i, j}}{\Delta t}-\frac{H_{x x} u_{i, j}}{(\Delta x)^{2}} .
$$

Inserting the indexed form (8) and (9) into the equality (10) and making some theoretical calculations, then the approach will be the $\Delta t \rightarrow 0$ and $\Delta x \rightarrow 0$. Therefore, the equality (10) will be same as left hand side of the Eq. (1). Thus, it can be seen that the Eq. (1) is consistent with FDM.

\subsection{Truncation error and stability analysis}

In this subsection, the stability and error analysis for the FDM are studied. For the stability, if there is a perturbation in the initial condition and then the small change would not cause the large error in the numerical solution. Simply, stability means that the scheme does not amplify errors and the error caused by a small perturbation in the numerical solution remains bound.

Theorem 1. The truncation error of the finite different method to the Eq. (1) is $(\Delta x)^{2}\left[O(\Delta t)^{2}+O(\Delta x)^{3}\right]$.

Proof. Inserting Eq. (4) and (5) into Eq. (1) gives

$$
\begin{aligned}
\frac{H_{t} u_{i, j}}{\Delta t}+O(\Delta t)^{2} & =\left(\frac{H_{x x} u_{i, j}}{(\Delta x)^{2}}+O(\Delta x)^{3}\right) \\
& -\left(u_{i, j}\right)^{3}+u_{i, j} .
\end{aligned}
$$

Inserting the equalities (2) and (3) into the Eq. (11) and do some necessary manipulations, then we obtain the following equality

$$
\begin{aligned}
u_{i+1, j}= & -u_{i-1, j}+u_{i, j}\left(2-(\Delta x)^{2}-\frac{(\Delta x)^{2}}{\Delta t}\right) \\
& +(\Delta x)^{2}\left(u_{i, j}\right)^{3}+\frac{(\Delta x)^{2}}{\Delta t} u_{i, j+1} \\
& +(\Delta x)^{2}\left(O(\Delta t)^{2}+O(\Delta x)^{3}\right) .
\end{aligned}
$$

Utilizing Eq. (12), one may write numerical solution $\hat{U}$ as

$$
\begin{aligned}
\hat{U}= & -u_{i-1, j}+u_{i, j}\left(2-(\Delta x)^{2}-\frac{(\Delta x)^{2}}{\Delta t}\right) \\
& +(\Delta x)^{2}\left(u_{i, j}\right)^{3}+\frac{(\Delta x)^{2}}{\Delta t} u_{i, j+1},
\end{aligned}
$$

and the truncation error $E$ as

$$
E=(\Delta x)^{2}\left[O(\Delta t)^{2}+O(\Delta x)^{3}\right] .
$$

Moreover, if $\Delta t$ and $\Delta x$ are considered as small as necessary, truncation error will be obviously very small. The limit of $E$ can be written as 


$$
\lim _{\substack{\Delta x \rightarrow 0 \\ \Delta t \rightarrow 0}} E=0 \cdot
$$

We can see that if $\Delta t$ and $\Delta x$ are configured for a value close to zero $\delta>0$, the following inequality is gotten

$$
|E|<\delta,
$$

which proves the stability of the FDM.

Theorem 2. The FDM in respect to the Cahn-Allen equation is unconditionally stable.

Proof. We consider the Von Neumann's Stability of the finite difference method for the Cahn-Allen. Let

$$
u_{i, j}=u(i \Delta x, j \Delta t)=u(p, q)=\varepsilon^{q} e^{I \xi p}, \xi \in[-\pi, \pi],
$$

where $p=i \Delta x, q=j \Delta t$ and $I=\sqrt{-1}$. Inserting Eq. (2), (3) and (13) into the equality (6), we can obtain

$$
\varepsilon \rightarrow 0 \text {, }
$$

According to the Von Neumann's Stability analysis [29], the FDM is stable if $|\varepsilon| \leq 1$. Hence, the FDM is unconditionally stable with the Cahn-Allen equation.

\section{4. $L_{2}$ and $L_{\infty}$ Error Norms}

To show how close the numerical approximations are close to the exact approximations the $L_{2}$ and $L_{\infty}$ error norms are utilized [30].

The $L_{2}$ error norm is defined as [30].

$$
L_{2}=\left\|u^{\text {exact }}-u^{\text {numeric }}\right\|_{2}=\sqrt{h \sum_{j=0}^{N}\left|u_{j}^{{ }^{\text {exact }}}-u_{j}^{\text {numeric }}\right|^{2}} \text {, }
$$

and $L_{\infty}$ error norm is defined as [30]

$$
L_{\infty}=\left\|u^{\text {exact }}-u^{\text {numeric }}\right\|_{\infty}=\operatorname{Max}_{j}\left|u_{j}^{\text {exact }}-u_{j}^{\text {numeric }}\right| \text {. }
$$

\section{Application}

In this section, we apply Finite Difference Method for Eq. (1) and consider numerical experiments. Recall the following hyperbolic function solution for Eq. (1) given in [1]:

$u_{1}(x, t)=-\frac{\left(3 A_{1}+\sqrt{9 A_{1}^{2}+24 c A_{0} B_{1}}\right)(-1+\operatorname{Tanh}[f(x, t)])}{6 A_{1}+2 \sqrt{9 A_{1}^{2}+24 c A_{0} B_{1}}-6 B_{1}(1+\operatorname{Tanh}[f(x, t)])}$,

where $f(x, t)=\frac{3 c_{1}-3 c t+\sqrt{2} c x}{4 c}$ and

$$
\mu \neq 0
$$$$
\frac{\left(3 A_{1}-3 B_{1}+\sqrt{9 A_{1}^{2}+24 c A_{0} B_{1}}\right)^{2}}{4 c^{2} B_{1}^{2}}
$$$$
-\frac{3\left(4 c A_{0} B_{1}+\left(A_{1}-B_{1}\right)\left(3 A_{1}+\sqrt{9 A_{1}^{2}+24 c A_{0} B_{1}}\right)\right)}{2 c^{2} B_{1}^{2}}>0 .
$$

If we put

$c=0.6, A_{0}=-3, B_{1}=-5, A_{1}=-1, c_{1}=0.1$, $0<x<1$ and $0<t<1$ for Eq. (14), the initial condition is

$u_{0}(x)=u(x, 0)=$

$$
-\frac{\left(3 A_{1}+\sqrt{9 A_{1}^{2}+24 c A_{0} B_{1}}\right)\left(-1+\operatorname{Tanh}\left[\frac{3 c_{1}+\sqrt{2} c x}{4 c}\right]\right)}{6 A_{1}+2 \sqrt{9 A_{1}^{2}+24 c A_{0} B_{1}}-6 B_{1}\left(1+\operatorname{Tanh}\left[\frac{3 c_{1}+\sqrt{2} c x}{4 c}\right]\right)},
$$

and under the above assumptions the exact solution of the Eq. (1) is as following

$$
u(x, t)=-\frac{12(-1+\operatorname{Tanh}[0.416667(0.3-1.8 t+0.848528 x)])}{24+30(1+\operatorname{Tanh}[0.416667(0.3-1.8 t+0.848528 x)])}
$$

Eq. (1) can be written as indexed with the help of finite difference operators

\begin{tabular}{|c|c|c|}
\hline$\Delta x=\Delta$ & $L_{2}$ & $L_{\infty} \quad 0.2$ \\
\hline 2.019 & $0^{-3}$ & $4.317 \times 10^{-3} 0.1$ \\
\hline 6.961 & $0^{-4}$ & $1.074 \times 10^{-3}$ \\
\hline 0.05 & $2.42301 \times 10^{-4}$ & $2.670 \times 10^{-4}$ \\
\hline 0.01 & $1.04962 \times 10^{-5}$ & $1.100 \times 10^{-5}$ \\
\hline
\end{tabular}

$u_{i+1, j}=-0.0001\left[10000 u_{i-1, j}-19999 u_{i, j}-u_{i, j}^{3}-100\left(-u_{i, j}+u_{i, j+1}\right)\right]$

A comparison of the obtained exact and numerical solutions are tabulate in Table 1.

Table 1. Numerical and exact solutions of equation (1) and absolute errors when $\Delta x=0.01$.

$\begin{array}{lllcc}\underline{x}_{i} & t_{j} \text { Numerical solution } & \text { Exact Solution } & \text { Absolute Error } \\ 0.00 & 0.01 & 0.184247 & 0.184258 & 1.06626 \times 10^{-5} \\ 0.01 & 0.01 & 0.183187 & 0.183197 & 1.06500 \times 10^{-5} \\ 0.02 & 0.01 & 0.182131 & 0.182142 & 1.06370 \times 10^{-5} \\ 0.03 & 0.01 & 0.181080 & 0.181091 & 1.06236 \times 10^{-5} \\ 0.04 & 0.01 & 0.180034 & 0.180044 & 1.06098 \times 10^{-5} \\ 0.05 & 0.01 & 0.178992 & 0.170900 & 1.05956 \times 10^{-5} \\ 0.06 & 0.01 & 0.177955 & 0.177966 & 1.05810 \times 10^{-5}\end{array}$

Table 2. $L_{2}$ and $L_{\infty}$ error norm when $0 \leq h \leq 1$ and $0 \leq x \leq 1$ 
Table2 shows that when $\Delta x$ and $\Delta t$ are small, the $L_{2}$ and $L_{\infty}$ error norm are decreasing. From Table 1-2 it is easily seen that results are in good agreement with the exact solution.
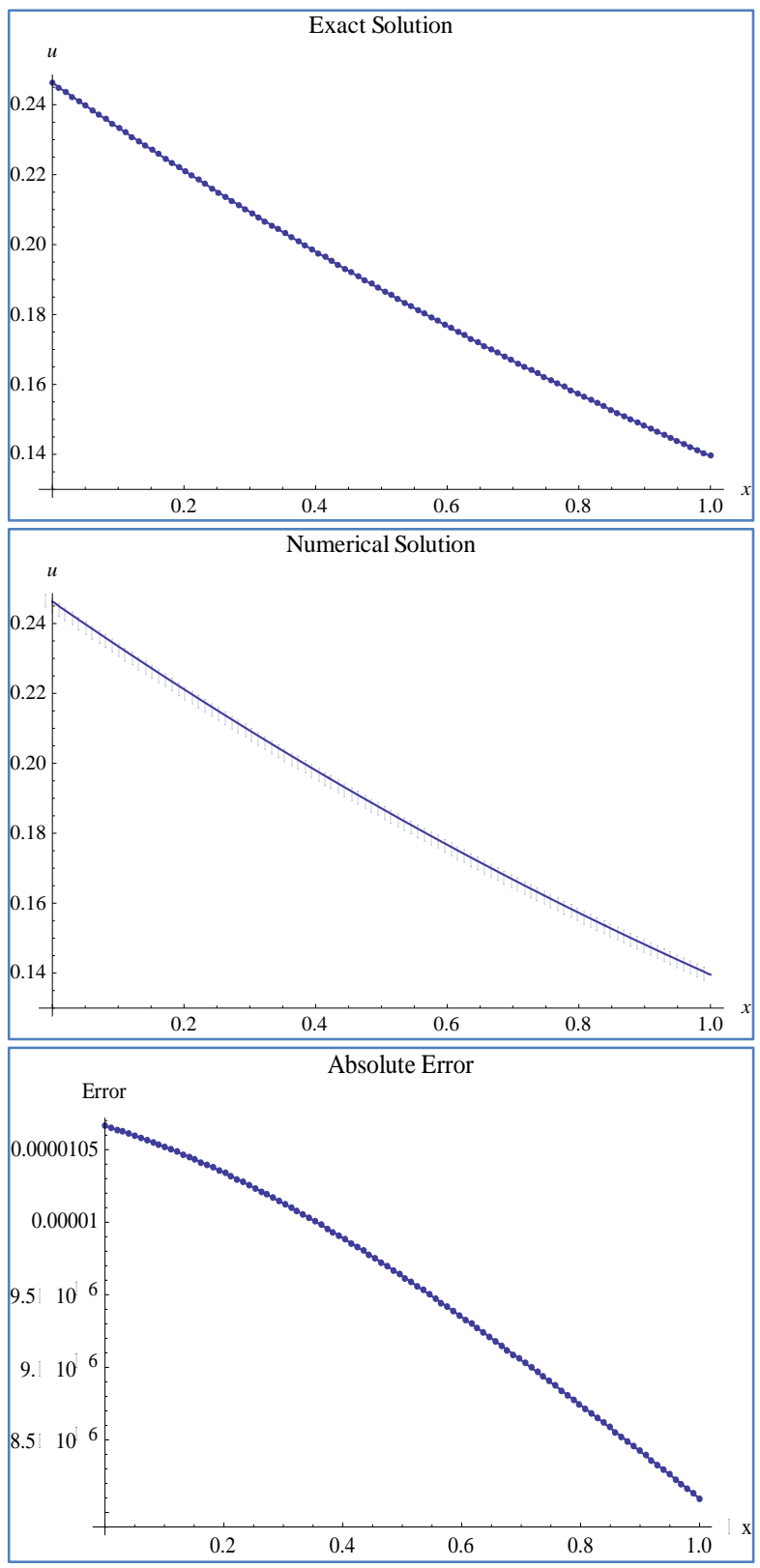

Figure 1. Numerical solution of Eq. (1) for finite difference method

Fig. 1 displays the physical behavior of the solution and shows that the exact approximations values are almost close to the numerically computed values. It is known that the truncation error depends on the choice of $\Delta x$ and $\Delta t$. Choosing the values to be very small gives rise to very small truncation error. This behavior of the numerical and exact solutions can be seen in the graphs above when the values of $\Delta x=\Delta t=0.01$.

\section{Remark}

The numerical results for example 1 have been obtained by using the programming language Wolfram Mathematica package. To the best of our knowledge, these numerical solutions have not been published previously, and these results are new numerical solutions for (1).

\section{Conculusion}

In this study, the FDM is used in approximating the numerical solutions to the Cahn-Allen equation. FDM is a useful numerical scheme for approximating the solutions of various nonlinear differential equations by defining suitable differential operators. The initial condition for the Cahn-Allen equation is obtained using the new analytical solution. The Cahn-Allen equation is written as indexed with the help of finite difference operators. Error analysis of the index equation was analyzed. Cahn-Allen equation is discussed with an example and error estimates obtained for the FDM. Furthermore, the behavior of potentials $u$ and absolute error are examined graphically.

\section{References}

[1] Bulut, H. (2017). Application of the modified exponential function method to the Cahn-Allen equation, AIP Conference Proceedings 1798, 020033.

[2] Villarreal, J. M. (2014). Approximate solutions to the allen-cahn equation using the finite difference method, Thesis, B.S., Texas A \& M International University.

[3] Xue, C. X., Pan, E. \& Zhang, S. Y. (2011). Solitary waves in a magneto-electro-elastic circular rod, Smart Materials and Structures, 20(105010), 1-7.

[4] Russell, J. S. (1844). Report on waves, 14th Mtg of the British Association for the Advancement of Science.

[5] Yang, Y. J. (2013). New application of the $\left(G^{\prime} / G, 1 / G\right)$-expansion method to KP equation, Applied Mathematical Sciences, 7(20), 959-967.

[6] Yokus, A. (2011). Solutions of some nonlinear partial differential equations and comparison of their solutions, Ph.D. Thesis, Firat University.

[7] Guo, S. \& Zhou, Y. (2011). The extended $\left(G^{\prime} / G\right)$ -expansion method and its applications to the Whitham-Broer-Like equations and coupled HirotaSatsuma KdV equations, Applied Mathematics and Computation, 215 (9) 3214-3221.

[8] Yokus, A. (2017). Numerical solution for space and time fractional order Burger type equation, Alexandria Engineering Journal, https://doi.org/10.1016/j.aej.2017.05.028.

[9] Yokus, A. \& Kaya, D. (2015). Conservation laws and a new expansion method for sixth order 
Boussinesq equation, AIP Conference Proceedings 1676, 020062 .

[10] Jawad, A. J. M., Petkovic, M. D. \& Biswas, A. (2010). Modified simple equation method for nonlinear evolution equations, Applied Mathematics and Computation, 217, 869-877.

[11] Su, L., Wang, W. \& Yang, Z. (2009). Finite difference approximations for the fractional advection-diffusion equation, Physics Letters A 373, 4405-4408.

[12] Odibat, Z. M. \& Shawagfeh, N. T. (2007). Generalized Taylor's formula. Applied Mathematics and Computation, 186 286-293.2.

[13] Liu, F., Zhuang, P., Anh, V., Turner, I. \& Burrage K. (2007). Stability and convergence of the difference methods for the space-time fractional advectiondiffusion equation, Applied Mathematics and Computation 191, 2-20.

[14] Su, L., Wang, W. \& Yang, Z. (2009). Finite difference approximations for the fractional advection-diffusion equation, Physics Letters A, 373, 4405-4408.

[15] Miura, M. R. (1978). Backlund transformation, Springer, Berlin.

[16] Motsa, S. S., Sibanda, P., Awad, F.G. \& Shateyi, S. (2010). A new spectral-homotopy analysis method for the MHD Jeffery-Hamel problem, Computers \& Fluids, 39(7), 1219-1225.

[17] Domairry, G., Mohsenzadeh, A. \& Famouri, M. (2009). The application of homotopy analysis method to solve nonlinear differential equation governing Jeffery-Hamel flow, Communications in Nonlinear Science and Numerical Simulation, 14(1), 85-95.

[18] Joneidi, A.A., Domairry, G. \& Babaelahi, M. (2010). Three analytical methods applied to Jeffery-Hamel flow, Communications in Nonlinear Science and Numerical Simulation, 15(11), 3423-3434.

[19] Alam, M. N., Hafez, M. G., Akbar, M. A. \& Roshid, H. O. (2015). Exact Solutions to the $(2+1)$ Dimensional Boussinesq Equation via exp $(\Phi(\eta))-$ Expansion Method, Journal of Scientific Research, 7(3), 1-10.

[20] Roshid, H. O. \& Rahman, Md. A. (2014). The exp $(-\Phi(\eta))$-expansion method with application in the (1+1)-dimensional classical Boussinesq equations, Results in Physics, 4, 150-155.

[21] Abdelrahman, M. A. E., Zahran, E. H. M. \& Khater, M. M. A. (2015). The exp $(-\phi(\xi))$-Expansion Method and Its Application for Solving Nonlinear Evolution Equations, International Journal of Modern Nonlinear Theory and Application, 4, 37-47.
[22] Baskonus, H. M., \& Bulut, H. (2015). On the complex structures of Kundu-Eckhaus equation via improved Bernoulli sub-equation function method. Waves in Random and Complex Media, 25(4), 720728.

[23] Bulut, H., Atas, S. S., \& Baskonus, H. M. (2016). Some novel exponential function structures to the Cahn-Allen equation. Cogent Physics, 3(1), 1240886 .

[24] Wang, M., Li, X., \& Zhang, J. (2008). The (G'G)expansion method and travelling wave solutions of nonlinear evolution equations in mathematical physics. Physics Letters A, 372(4), 417-423.

[25] Feng, J., Li, W., \& Wan, Q. (2011). Using G' Gexpansion method to seek the traveling wave solution of Kolmogorov-Petrovskii-Piskunov equation. Applied Mathematics and Computation, 217(12), 5860-5865.

[26] Yokus, A., Baskonus, H. M., Sulaiman, T. A., \& Bulut, H. (2018). Numerical simulation and solutions of the two-component second order $\mathrm{KdV}$ evolutionarysystem. Numerical Methods for Partial Differential Equations, 34(1), 211-227.

[27] Şener, S. Ş., Saraç, Y., \& Subaşı, M. (2013). Weak solutions to hyperbolic problems with inhomogeneous Dirichlet and Neumann boundary conditions. Applied Mathematical Modelling, 37(5), 2623-2629.

[28] Subaş1, M., Şener, S. Ş., \& Saraç, Y. (2011). A procedure for the Galerkin method for a vibrating system. Computers \& Mathematics with Applications, 61(9), 2854-2862.

[29] Rezzolla, L. (2011). Numerical methods for the solution of partial differential equations. Lecture Notes for the COMPSTAR School on Computational Astrophysics, 8-13.

[30] Yokus, A., \& Kaya, D. (2017). Numerical and exact solutions for time fractional Burgers' equation. Journal of Nonlinear Sciences and Applications, 10(7), 3419-3428.

Asıf Yokus is currently doctor of Actuary in Firat University. His research interests include finite different method, collocation method, analytical methods for nonlinear differential equations and numerical solutions of the partial differential equations.

Hasan Bulut is currently professor of Mathematics in Firat University. His research interests include stochastic differential equations, fluid and heat mechanics, finite element method, analytical methods for nonlinear differential equations and numerical solutions of the partial differential equations. 
An International Journal of Optimization and Control: Theories \& Applications (http://ijocta.balikesir.edu.tr)

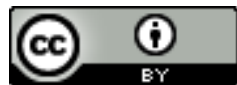

This work is licensed under a Creative Commons Attribution 4.0 International License. The authors retain ownership of the copyright for their article, but they allow anyone to download, reuse, reprint, modify, distribute, and/or copy articles in IJOCTA, so long as the original authors and source are credited. To see the complete license contents, please visit http://creativecommons.org/licenses/by/4.0/. 